\title{
An Analysis of Sentimental Data using Machine Learning Techniques
}

\author{
Surbhi Chandhok \\ Bachelors of technology \\ Computer Science \\ Galgotias College of \\ Engineering \& \\ Technology \\ Uttar Pradesh, India
}

\author{
Romil Anand \\ Bachelors of \\ Technology \\ Computer Science \\ Galgotias College of \\ Engineering \& \\ Technology \\ Uttar Pradesh, India
}

\author{
Soumay Gupta \\ Bachelors of \\ Technology \\ Computer Science \\ Galgotias College of \\ Engineering \& \\ Technology \\ Uttar Pradesh, India
}

\author{
Aatif Jamshed \\ Masters of Technology \\ Computer Science \\ Galgotias College of \\ Engineering \& \\ Technology \\ Uttar Pradesh, India
}

\begin{abstract}
Sentiment analysis, basically, comprises of identifying different opinions or emotions in source text and then classifying them in its accordance. Social media generates extensive sentiment rich dataset in the form of Social media in through status updates, tweets, short video clips, blog posts etc. At the same time, it is relatively more difficult to do a Twitter estimation investigation when contrasted with general assessment examination. This is because of the presence of foul words, slang language and incorrect spellings. The maximum character limit per tweet in Twitter is 140 . There are two strategies used to analyse sentiments, emotions and/or opinions from the source file. These two strategies are:
\end{abstract}

\section{1) Approach based on knowledge \\ 2) Approach based on learning of machine.}

Throughout this paper, we've tried to analyse the posts of twitter related to a wide range of electronic products such as mobiles, laptops, video games television sets etc. using Machine Learning approach. During the time spent doing notion investigation in a specific area, it is conceivable to distinguish and make sense of the impact of space data as per the estimation order. We've additionally presented another element vector, in order to just group the tweets as plain constructive, pessimistic and infer people groups' feeling about items.

\section{Keywords}

Twitter, Sentiment Analysis, Machine Learning Techniques

\section{INTRODUCTION}

The advanced time of Internet has altogether changed the way individuals express their sentiments. The most recent form of expressing oneself, these days, is through blog posts, product review websites, online discussion forums etc. It has been found that people, to a great extent, heavily rely upon this user generated data. For example- when somebody needs to purchase any item, they will begin to look into its surveys online before going to a ultimate conclusion. Since the measure of this client created substance is far too substantial, it gets greatly several sentiment analysis techniques have been put into application, so as to automate this.

Two major techniques used in this analysis are "Symbolic techniques or Knowledge base approach" and "Machine learning techniques". For the approach based on knowledge , a huge database of predefined emotions along with an effective knowledge representation for identifying sentiments, is needed. Approach based on leaning of machine, primarily, puts into utilization, a preparation set that builds up a slant classifier, which additionally orders sentiments\& assessments. Since a predefined database of entire sentiments is not required for "machine learning approach", it is ideally more clear and more straightforward than the "Knowledge base" approach. In this paper, we should run over and talk about unmistakable machine learning strategies for orchestrating tweets.

The analysis of sentiments and opinions is generally led at various levels differing from coarse level to fine level. Coarse level sentiment examination oversees choosing the estimation of an entire document and Fine level oversees quality level conclusion investigation. Sentence level sentiment investigation comes in the middle of two [1]. Several kinds of researches on the division of sentiment analysis of user based surveys. Past research has demonstrated that the exhibitions of assumption classifiers are subject to themes. In light of that, we can't state that one classifier is the best for all points since one classifier doesn't reliably beats the other. Sentimental Analysis in twitter can be very troublesome because of its short length of maximum 140 characters in a tweet. Nearness of changed emoticons, foul words and mistaken spellings in tweets constrain to have a pre- handling wander before highlight removal. There are diverse component taking out techniques to gather pertinent elements from content which can be connected to tweets too. Be that as it may, the component extraction is to be done in two stages to separate important elements. In the primary stage, twitter particular elements are separated. At that point, these elements are expelled from the tweets to make ordinary content. From that point onward, again highlight extraction is done to get more components. This is the thought utilized as a part of this paper to create an effective component vector for dissecting twitter supposition. Since no standard dataset is accessible for twitter posts of electronic gadgets, we made a dataset by gathering tweets for a specific time frame.

By doing opinion analysis on a particular space, it is conceivable to distinguish the impact of area data in picking an element vector. Diverse classifiers are utilized to do the grouping to discover their impact in this specific area with this specific element vector. 


\section{RELATED WORK}

There are two essential systems to identify assumptions from content. They are predefined procedures and Machine Learning systems[2]. The following two areas manage such methods.

\subsection{Typical Techniques}

A significant part of examination in unsupervised opinion characterization utilizing typical strategies makes utilization of accessible lexical assets. Turney [3] used pack of-words approach for the opinion examination. In that approach, associations $\mathrm{b} / \mathrm{w}$ the individual words are not considered \& the record is addressed as an immaterial aggregation of words. To decide a general conclusion, assessments of each word is resolved \& those qualities are consolidated with some total capacities. He found the extremity of a survey in view of the normal semantic introduction of the tuples extricated from the audit where tuples are expressions which have descriptive words, modifiers. He found a semantic introduction of the tuples utilizing a web crawler Altavista.

Kamps et al[4] utilized the database which is lexical Word Net [5], in order to decide a passionate substance of the word with various measurements. They built up a separation metric on Word Net and decided the semantic introduction of descriptive words. Word Net type database comprises of different words associated by equivalent word relations. Baroni et al[6] built up the framework utilizing word space display formalism that beats the trouble in the substitution by lexical errand. It speaks to a neighbourhood setting of the word alongside it is general circulation. Balahur et al[7] presented Emoti Net, a calculated representation of content that stores the structure and the semantics of genuine occasions for a particular area. Emoti net utilized the idea of Automata of Finite State to distinguish a passionate reactions activated by activities. One member of Sem Eval 2007 Task No. 14 [8], utilized coarse grained and the fine grained ways to deal with recognized opinions in the news features. In coarse grained approach, they performed matched gathering of assessments and in fine grained approach they requested feelings into different levels.

Information base approach is observed to be troublesome because of the prerequisite of an immense lexical database. Since interpersonal organization produces immense measure of information consistently, some of the time bigger than the span of accessible lexical database, assessment examination got to be distinctly dull and mistaken.

\subsection{Techniques of Machine Learning}

Strategies of learning by machine utilize a preparation set and a test set for characterization. Preparing sets containing input highlight vectors and marks of compared classes. Utilizing this preparation set, a grouping model is created which tries to order the info highlight vectors into comparing class marks. By then, a test set is used to support the model by envisioning the class names of subtle component vectors. By doing feeling examination on a specific space, it is possible to recognize the effect of region information in picking a component vector. Diverse classifiers are utilized to do the grouping to discover their impact in this specific area with this specific element vector. Various $\mathrm{m} / \mathrm{c}$ learning procedures like Naïve Bayes (NB), Max Entropy (ME), and Vector Support Machines (VSM) are utilized to characterize audits[9]. A portion of components that can utilize for estimation grouping are Term Presence, Term Frequency, nullification, n grams \& Part-ofthe- Speech[1]. These elements are utilized to discover the semantic introduction of words, expressions, sentences \& of reports. Semantic introduction is an extremity which might be either +ve or -ve.

Domingos et al[10] found Naive Bayes functions admirably for a specific issues with an exceedingly subordinate components. This is amazing as a fundamental presumption of Naive Bayes is that the components are autonomous. Zhen Niu et al.[11] presented another model in ia which productive methodologies which utilize for highlighting determination, weight calculation \& arrangement. A new model depends on the Bayesian calculation. Here weights of classifier are balanced by the making utilization to agent highlight \& remarkable element. 'Assign highlight' is the information that addresses a class and 'Phenomenal component' is the information that gives help in perceiving classes. Using those weights, they found out the probability of each request and along these lines upgraded the Bayesian estimation.

Barbosa et al.[12] planned a 2-stage programmed conclusion examination technique for arranging tweets. They utilized a loud preparing set to diminish the naming exertion in creating classifiers. Firstly, that they assembled tweets into the subjective and target tweets. From such point forward, subjective tweets are delegated +ve \& -ve tweets. Celikyilmaz et al. [13] built up an articulation based on word bunching technique for normalizing uproarious tweets. In articulation based on word bunching, words that have comparative elocution are grouped \& doled out normal tokens. They additionally utilized content preparing procedures like allotting comparative tokens for the numbers, html joins, client identifiers \& target association names for standardization. In the wake of doing standardization, they utilized probabilistic models to distinguish extremity dictionaries. They performed characterization utilizing a BoosTexter classifier with such extreme vocabularies as elements \& acquired the decreased mistakerate.

$\mathrm{Wu}$ et al proposed an impact likelihood demonstrate for the twitter notion investigation. On the off chance that @ NameOfUser is found in the body of a tweet, it is affecting activity \& it adds to impacting likelihood.

A tweet that starts with @NameOfUser is basically a retweet that speaks to an affected activity and it adds to impacted likelihood. They watched that there is the solid relationship $\mathrm{b} / \mathrm{w}$ such probabilities.

Pak et al. made a twitter-corpus via naturally gathering tweets utilizing Twitter API \& consequently commenting on those utilizing various emoticon. Utilizing that corpus, they manufactured the conclusion classifier in view of a multinomial Naive Bayes classifier that utilizations $\mathrm{N}$ gram and PS-labelsas components. In this strategy, there is the shot of blunder since sentiments and emotions of tweets in preparing set which are named exclusively in view of the extremity of emoticons. The preparation set is additionally less effective since it is then just contains tweets which have emoticons.

\section{SUGESSTED ALTERNATE}

One can make a dataset consisting of twitter posts about electronic items. Tweets are basically short and crisp messages with excess usage of slang and incorrect spellings. Basically, we play out a sentiment analysis on the basis of each sentence. This process is carried out in three main stages. In the initial first stage, pre-processing is finished. At that point, an element vector is made utilizing pertinent elements. At long last utilizing varied tweets, classifiers etc. are ordered into two classes- positive $\&$ negative. 
In light of amount of several tweets in almost every class, a final opinion is determined.

\subsection{Formation of the Dataset}

Table 1. Insights Used Through A Dataset

\begin{tabular}{|l|l|l|l|}
\hline $\begin{array}{l}\text { Random } \\
\text { Dataset }\end{array}$ & Positivity & Negativity & Sum up \\
\hline Training & 1000 & 1000 & 2000 \\
\hline Testing & 200 & 200 & 400 \\
\hline
\end{tabular}

As the rudimentary dataset of twitter isn't accessible to the electronic items area, another dataset was made by gathering numerous tweets over a timeframe running from April 2013 to May 2013. Tweets are compiled naturally by utilizing the Twitter's API and therefore, they still are physically commented on as either positive or negative. One can make dataset by combining 600 highly positive tweets and other 600 highly negative tweets. In the above Table-1, it is demonstrated exactly how we segregate a dataset into one preparing set and other test set.

\subsection{Tweets under Processing}

Due to the incorrect spellings and slangs, Catchphrase extraction is not very smooth or convenient. So to keep away from this, we perform a pre-processing step before highlight extraction. Preliminary steps incorporate expelling your URL and thus, keeping away from incorrect spellings and slang words. Incorrect spellings are evaded as the replacement of the characters if repetition with 2 or more occurrences takes place. Slang words contribute significantly to a tweet's feeling. So one can't essentially expel those slangs. Accordingly, a slang lexicon is kept up so as to interchange with the used slang word happening to several tweets, incorporated with there related implications. Space data contributes much to development of the slang lexicon.

\subsubsection{Process of Making Vector Featuring}

Highlight picking out is carried mainly in two basic stages. Initially, extraction of varied twitter specific components takes place. The applicable components specific to twitter are Hashtags \& emoticons. It is observed that emoticons can either be affirming and positive or discouraging and negative. Therefore, distinctive weights are given to them. There might as well be either or both of the positive and negative hash tags. Hence, the total number of positive as well as negative hash tags are included as two different components in an element vector featuring.

Components specified to Twitter may not available in every tweets. Thus, it's imperative that a further component extraction is done to acquire different elements. In the wake of separating twitter particular elements, they are expelled from the tweets. Tweets can be then considered as straightforward content.

Numbers of +ve \& -ve catchphrases among several tweets are utilized as two distinct components in the element vector. Active presence of nullification contribute much to the estimation. So their presence, in this scenario, is additionally included as a pertinent element.

Every watchwords can't be dealt with in the same manner, within the sight of different positive and negative catchphrases. Consequently a unique catchphrase is chosen fromevery one of the tweets. On account of tweets having just positive catchphrases or just negative watchwords, an inquiry is done to recognize a watchword having important grammatical form. A significant grammatical feature is descriptive word, qualifier or verb. Such a pertinent grammatical feature is characterized in light of their significance in deciding assumption. Catchphrases that are a descriptive word, intensifier or verb demonstrates relatively more emotional or sentimental than the others. On the off chance that an applicable grammatical feature can be resolved for a catchphrase, then that is taken as an uncommon watchword. Generally, a catchphrase is chosen arbitrarily from the accessible watchwords as unique catchphrase. In the event that positive along with negative watchwords are available in a tweet that if we select any catchphrase having pertinent grammatical feature. In the event that pertinent grammatical form is available for both positive and negative catchphrases, none of them is picked. An exceptional catchphrase highlight is given a weight of "1" on the off chance, that it is certainly positive, and '- 1 ' on the off chance that it is negative and "0" in its nonappearance. Grammatical form highlight is given an estimation of "1" in the event that it is significant and " 0 " generally.

In this manner, highlight vector is made out of 8 important. The 8 highlights utilized are grammatical form (pos) label, uncommon catchphrase, dynamic nearness of nullification, emoticon, the quantity of +ve watchwords, the quantity of -ve catchphrases.

\subsection{Classifying Sentiments}

In the wake of making a segment vector, a course of action are done using Naive Bayes, Vector Support Machine, Maximum Entropy \& Ensemble classifiers \& their executions are investigated.

\section{ARRANGEMENT METHODS \& TECHNIQUE}

There are distinctive sorts of classifiers that are by and large utilized for the content arrangement which can be additionally built-in for twitter opinion characterization.

\subsection{Naive Bayes Classifier}

Naive Bayes Classifier make the utilization of considerable number of components in an element vector and breaks them down exclusively as are similarly free of each other. The restrictive likelihood for Naive Bayes can be characterized as

$$
\mathrm{P}(\mathrm{Z} \mid \mathrm{cj})=\Pi \mathrm{mi}=1 \mathrm{P}(\mathrm{zi} \mid \mathrm{cj})
$$

"Z" is an element vector characterized as $\mathrm{Z}=\{\mathrm{z} 1, \mathrm{z} 2, \ldots . \mathrm{zm}\} \&$ cj is a class name. Here, in our work, there are distinctive free elements like emoticons, enthusiastic watchword, number of positive and negative catchphrases, and tally of +ve \& -ve hash labels which are viably used by the classifier of Naive Bayes for grouping. This algorithm does not consider the connectionsb/w elements. So it can't use the connections b/w grammatical form tags, passionate catchphrase \& refutation.

\subsection{VSM Classifier}

VSM Classifier utilizes substantial edge of characterization. It isolates tweets utilizing the hyper plane. VSM utilizes the the discriminative capacity characterized :

$$
a(Z)=w T \varphi(Z)+b
$$


"Z" as element vector, "w" as weights vector and "b" as predisposition vector. $\varphi()$ is non straight map from information space to top dimensional element spaces. "w" \& "b" are found out consequently on preparation set. Here we utilized the direct portion for order. It keeps up the wide crevice b/w two classes.

\subsection{Greatest Entropy Classifier}

In Max Entropy Classifier,not even a single suspicions are taken with respect to the relationship b/w elements. The classifier dependably tries to augment the entropy of the framework by evaluating a restrictive appropriation of the class mark. The contingent circulation is characterized :

$$
\mathrm{P} \lambda(\mathrm{c} \mid \mathrm{Z})=1 / \mathrm{X}(\mathrm{Z}) \text { expo }(\mathrm{Z} \text { i } \lambda \text { ifi }(\mathrm{Z}, \mathrm{c}))
$$

"Z" is component vector \& "c" is our class name. $\mathrm{X}(\mathrm{Z})$ is the standardization component and $\lambda \mathrm{i}$ is the weight coefficient. fi( $\mathrm{Z}, \mathrm{c})$ is component work which is characterized :

$$
\begin{aligned}
& \text { fi }(Z, c)=\operatorname{if}(f i(Z, c)==1) Z=Z i \& c \\
& =c i \quad \text { (4) else, } 0
\end{aligned}
$$

In our component vector, the connections between grammatical feature tag, enthusiastic catchphrase and nullification are used successfully for order.

\subsection{Group classifier}

Group classifiers are of various sorts. They attempt to ensure utilization of an elements of all base classifiers to do there best grouping.

Here a troupe classifying technique is produced by the voting principle. These classifier are going to characterize in light of the yield of greater part of classifiers.

\section{EVALUATION}

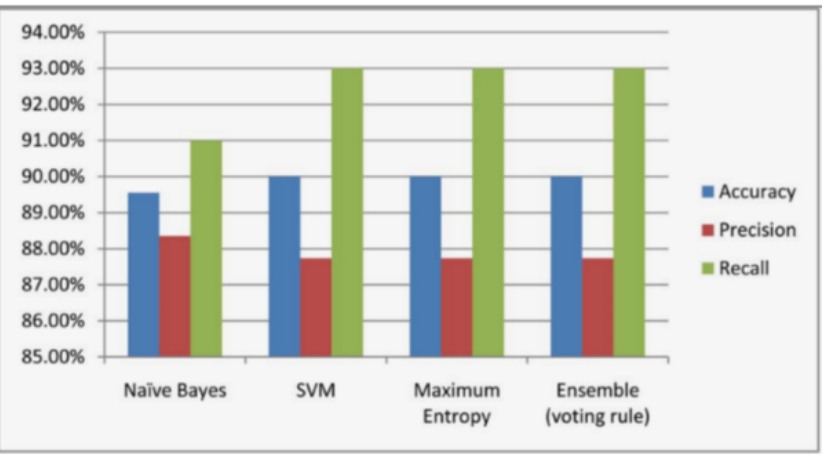

Fig. 1. Execution of Various kinds of classifiers used in Sentiment Analysis of Twitter

Utilizing Twitter API, tweets identified with items are gathered.

The set of Data is made utilizing 1200(approx.) twitter posts of e- Items. Dataset is part into a preparation set of about 1000 tweets and a test set of 200 tweets (approx). We utilized Stanford postager to remove grammatical feature tags from twitter tweets.

Since we chosen item space, their is no need of examining subjective \& target tweets independently. To distinguish item's nature, both of the qualities contribute correspondingly. It depicts in what way the setting or space data influences assessment investigation. These classifiers are tried utilizing Mat lab test system. We put into use three sorts of essential classifiers IVSM, Max Entropy, Naive
Bayes \} \& group classifier for assumption arrangement. SVM \& classifiers of Naive Bayes are actualized utilizing Mat lab worked in capacities. Greatest Entropy which classifies the actualized putting in application MaxEnt software2. Execution of these classifiers has appeared in Figure 1. Every one of these classifiers has practically comparable execution.

\section{CONCLUSIONS}

We have diverse Symbolic as well as Machine Learning strategies to recognize estimations from content. Machine Learning procedures are less complex and proficient then predefined systems. Such systems are connected for the assumption of twitter investigation. Their can be issue in managing distinguishing passionate catchphrase from tweets having numerous watchwords. It is additionally hard to deal with incorrect spellings \& words slang. For managing such issue, a proficient element vector is made for highlight removal technique from two stages after appropriate preliminary procedure. In the initial step, twitter particular elements are separated and added to the element vector. From the same point forward, such elements are expelled through tweets and then again include picking up technique is done though it is on typical content. Such components likewise summed up with an element vector. Every one in these classifiers have practically comparable precision for the latest vector component. This vector component is carried out perfectly well for all customized electronicscategory.

\section{ACKNOWLEDGMENT}

This research would not have been completed without the guidance of Mr. Manish Kumar Sharma (Project Coordinator) and HOD CSE Dr. Bhawna Mallick. We would like to thank all the professors who guide us in dis project.

\section{REFERENCES}

[1] Y. Mejova, "Sentiment analysis: An overview," Comprehensive exam paper, available on http://www.cs.uiowa.edu//ymejova/publications/Comps YelenaMejova. pdf [201002- 03], 2009.

[2] P. D. Turney, "Thumbs up or thumbs down?: semantic orientation applied to unsupervised relegation of reviews," in Proceedings of the 40th annual meeting on sodality for computational linguistics, pp. 417-424, Sodality for Computational Linguistics, 2002.

[3] E. Boiy, P. Hens, K. Deschacht, and M.-F. Moens, "Automatic sentiment analysis in on-line text," in Proceedings of the 11th International Conference on Electronic Publishing, pp. 349-360, 2007.

[4] J. Kamps, M. Marx, R. J. Mokken, and M. De Rijke, "Using wordnet to quantify semantic orientations of adjectives," 2004.

[5] C. Fellbaum, "Wordnet: An electronic lexical database (language, verbalization, and communication)," 1998.

[6] D. Pucci, M. Baroni, F. Cutugno, and A. Lenci, "Unsupervised lexical supersession with a word space model," in Proceedings of EVALITA workshop, 11th Congress of Italian Sodality for Artificial Astuteness, Citeseer, 2009

[7] A. Balahur, J. M. Hermida, and A. Montoyo, "Building and exploiting emotinet, an erudition base for emotion detection predicated on the appraisal theory model," Affective Computing, IEEE Transactions on, vol. 3, no. 1, pp. 88-101, 2012. 
[8] C. Strapparava and R. Mihalcea, "Semeval-2007 task 14 Affective text," in Proceedings of the 4th International Workshop on Semantic Evaluations, pp. 70-74, Sodality for Computational Linguistics, 2007.

[9] G. Vinodhini and R. Chandrasekaran, "Sentiment analysis and opinion mining: A survey," International Journal, vol. 2, no. 6, 2012.

[10] L. Barbosa and J. Feng, "Robust sentiment detection on twitter from partial and strepitous data," in Proceedings of the 23rd International Conference on Computational Linguistics: Posters, pp. 36-44, Sodality for Computational Linguistics, 2010.

[11] Z. Niu, Z. Yin, and X. Kong, "Sentiment relegation for microblog by machine learning," in Computational and
Information Sciences ( ICCIS), 2012 Fourth International Conference on, pp. 286-289, IEEE, 2012.

[12] P. Domingos and M. Pazzani, "On the optimality of the simple bayesian classifier under zero-one loss," Machine Learning, vol. 29, no. 2-3 , pp. 103-130, 1997.

[13] A. Celikyilmaz, D. Hakkani-Tur, and J. Feng, "Probabilistic model-predicated sentiment analysis of twitter messages," in Verbalized Language Technology Workshop (SLT), 2010 IEEE, pp. 79-84, IEEE, 2010.

[14] Y. Wu and F. Ren, "Learning sentimental influence in twitter," in Future Computer Sciences and Application (ICFCSA), 2011 International Conference on, pp. 119122, IEEE, 2011 